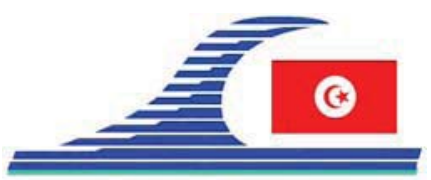

Conférence Méditerranéenne Côtière et Maritime EDITION 1, HAMMAMET, TUNISIE (2009)

Coastal and Maritime Mediterranean Conference

Disponible en ligne - http://www.paralia.fr - Available online

\title{
Le Shadows, un sonar de haute précision permettant une cartographie marine
}

\section{Eric DELORT ${ }^{1}$}

1. iXsurvey, 46 quai Mitterrand, 13600 La Ciotat, France.

eric.delort@ixsurvey.com

\section{Résumé :}

Shadows est le nouveau système sonar d'IXSEA (site web). Composé de deux sonars latéraux et d'un sonar frontal, Shadows permet de couvrir intégralement $600 \mathrm{~m}$ de fauchée avec une résolution constante sur les côtés de $15 \mathrm{~cm}$ et une résolution de $50 \mathrm{~cm}$ au nadir. Ce système utilise toutes les compétences d'IXSEA et d'IXSURVEY : acoustique, électronique, informatique, positionnement, imagerie, ... .

Le résultat de ce travail est le seul sonar commercial utilisant la technologie d'antenne synthétique et c'est aussi le seul sonar proposant un "Gapfiller» (système de positionnement sous-marin). L'intérêt pour IXSURVEY d'utiliser un Shadows réside dans la large fauchée et surtout dans la possibilité de localiser le poisson et donc d'obtenir des cartes très précises. Cet outil très performant permet la réalisation de campagnes de sondage cartographique pour d'importantes superficies.

Les principales opérations nécessitant l'utilisation du Shadows sont :

- les cartographies d'intérêt patrimonial (localisation et suivi d'herbes marines protégées en Méditerranée),

- les cartographies de récifs artificiels et le suivi des structures dans le temps,

- les cartographies biosédimentaires pour des travaux côtiers et/ou offshore (aménagement de ports, de champs éoliens...).

\section{Mots-clés :}

Sonar à balayage latéral - Cartographies biosédimentaires - Antenne synthétique Gapfiller - Shadows

\section{Introduction}

Les sonars à balayage latéral sont usuellement mis en œuvre pour cartographier les fonds marins, depuis les zones côtières, jusqu'aux zones plus profondes.

Un sonar est un imageur acoustique du fond, constitué d'une centrale embarquée et d'un câble tractant un poisson. Ce poisson génère classiquement un faisceau étroit permettant une imagerie des fonds.

Or, de nombreuses inconnues entrainent des erreurs dans la cartographie obtenue :

- l'altitude et la position du poisson sont inconnues,

- la perte de la géométrie des informations,

$$
\text { DOI: } 10.5150 / \mathrm{cmcm} .2009 .069-5
$$


- l'absence d'information au nadir (pas d'ombre à la verticale),

- la compression de l'information.

En outre, les problèmes de divergence acoustique entraînent un pouvoir de résolution variable avec la distance et une déformation de l'aspect. Les soucis de résolution des images sont fonction de la vitesse et de la fréquence. Par ailleurs, plus haute est la fréquence, meilleure est la résolution, mais plus faible la portée du sonar.

Enfin, plus la longueur de câble filée est importante, plus l'imprécision augmente pour le positionnement des éléments sous-marins.

Les images de la figure 1 illustrent la précision de l'imagerie sonar en fonction de la vitesse du bateau. Les opérations de sondage cartographique demandent des appareils :

- avec une meilleure résolution (quelques centimètres),

- avec moins de distorsion dans les images,

- avec une cartographie en temps réel,

- avec une gestion et une exportation des données plus facile,

- intégrant des données dans des systèmes d'informations géographiques,

- avec un meilleur taux de couverture horaire.

\section{Les objectifs du système Shadows}

Le but de la mise au point de ce nouveau sonar est de répondre aux exigences techniques et cartographiques sous-marines, soit :

- compenser la divergence acoustique pour une meilleure qualité des images,

- supprimer le gap (zone non lue) au nadir : optimisation des temps de mesures,

- fournir des images géoréférencées et ortho-rectifiées, comparable à l'imagerie satellitaire.

Les éléments techniques apportés correspondent en première approche à connaître la trajectoire 3D du poisson lorsqu'il est tracté. Pour cela, une centrale inertielle (PHINS + DVL) et un système de positionnement sous-marin (GAPS) ont été installés à bord du poisson.
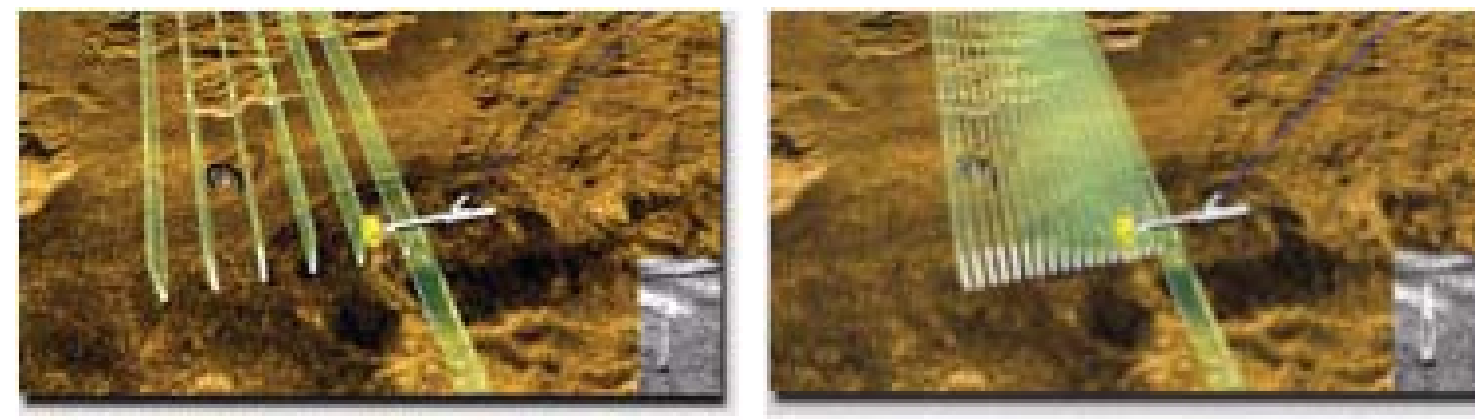

Figure 1. Exemple de différence de vitesse du bateau et de qualité d'acquisition. 
Le couplage du DVL permet une dérive de 1'ordre de $2 \mathrm{~mm} \cdot \mathrm{s}^{-1}$, une position à court terme, et une mesure de l'altitude (application des corrections en temps réel).

Le problème intervient lors d'une longue période de mesures sans arrêt: sur $24 \mathrm{~h}$ d'utilisation en continu, l'acquisition présente une dérive de $200 \mathrm{~m}$.

Pour pallier cette dérive, un système USBL de type positionnement sous-marin GAPS vient compléter l'acquisition et bloquer la dérive de la PHINS.

Ce système complet (PHINS + DVL + GAPS) permet un positionnement très précis en 3D du poisson, et donc une grande précision dans la cartographie sous-marine.

Le système est complété à bord par l'installation d'un GPS, permettant de donner la position initiale à la centrale inertielle.

La couverture du nadir est réalisée par l'installation d'un sonar frontal sur le poisson (voir figure 2). Ce sonar possède 4 émetteurs à $300 \mathrm{kHz}, 48$ récepteurs, une portée de 20 à $80 \mathrm{~m}$ en fonction de l'altitude du poisson et une résolution entre 15 et $40 \mathrm{~cm}$. Cette couverture au nadir permet d'optimiser le passage du bateau et de réduire les temps de navigation tout en augmentant la surface couverte lors de l'acquisition. Le cliché de la figure 3 présente le poisson Shadows tracté en surface derrière le bateau.

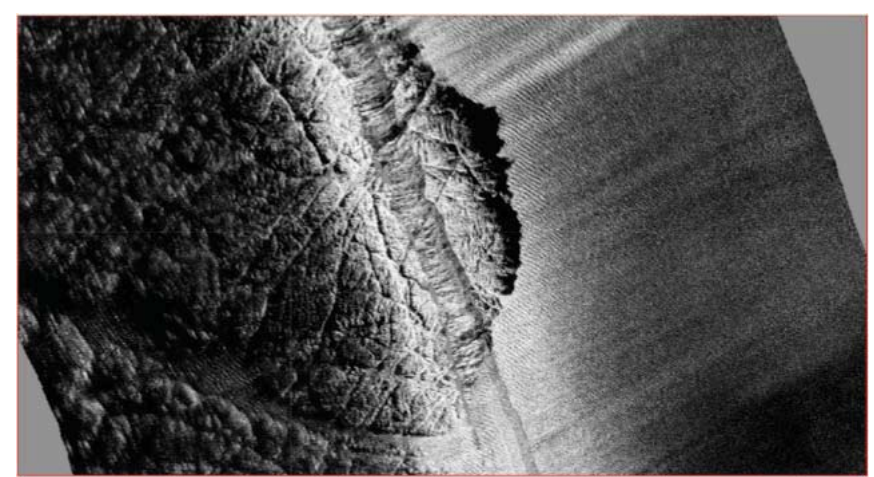

Figure 2. Image acoustique du Shadows - le sonar frontal permet de visualiser les échos au nadir.

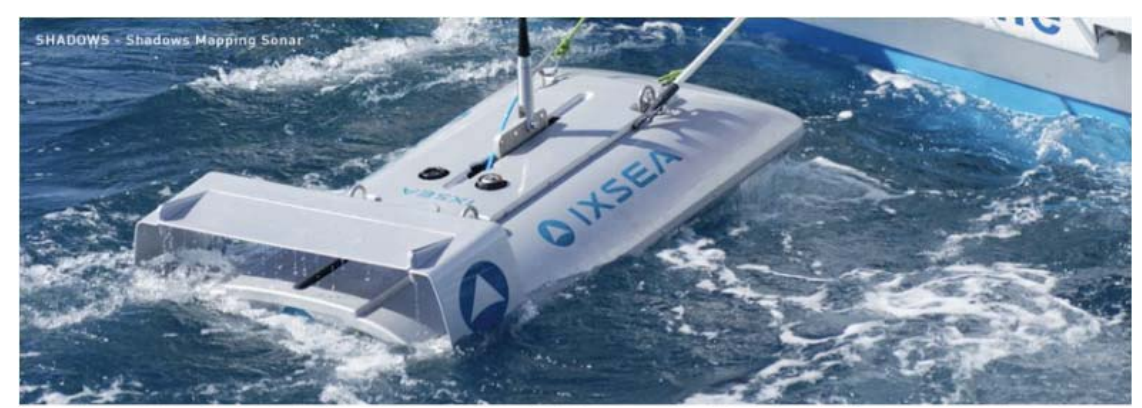

Figure 3. Poisson Shadows tracté derrière le bateau.

\section{Les opérations de sondage cartographique à l'aide du système Shadows}

Ce nouveau sonar permet donc :

- un positionnement précis du poisson lors des acquisitions, 
- une large fauchée $(600 \mathrm{~m})$,

- une très bonne résolution $(15 \mathrm{~cm})$.

Lors des acquisitions en mer, les éléments inertiels à bord du poisson permettent une mesure par mer formée 4 à 5 , alors qu'un sonar «normal » n'est utilisable que jusqu'à un état de mer de 3 à 4 . Les jours de mesures sont donc optimisés.

Le post-traitement des données collectées lors de l'acquisition est également diminué comparativement à celui d'un sonar classique. En effet, le temps nécessaire de posttraitement des mosaïques du Shadows est 3 fois moins long que pour un sonar classique. Le cliché de la figure 4 est une mosaïque sonar et correspond à plusieurs lignes passées à l'aplomb des champs de récifs artificiels à Marseille. La forme générale du plan d'aménagement en triangle est très visible. La longueur des ombres portées de chaque unité permet de calculer leur hauteur.

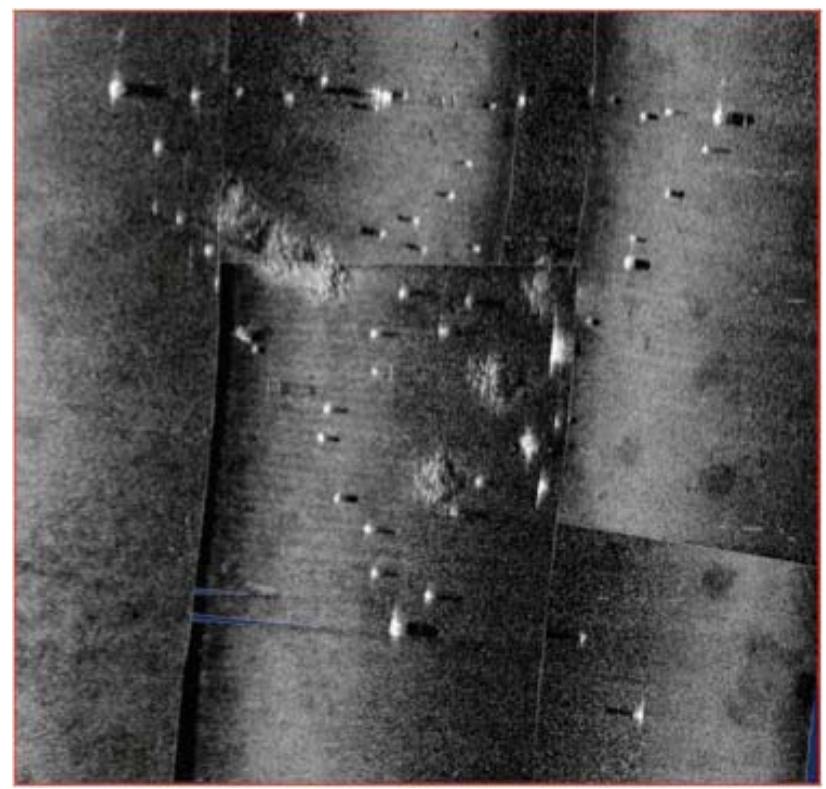

Figure 4. Aménagement en récifs artificiels à Marseille : mosaïque sonar.

\section{Conclusions et perspectives}

Ce type d'appareil permet un travail plus rapide, une meilleure qualité des images, un meilleur géo-référencement des données acquises et une mosaïque en temps réel permettant un post-traitement limité. L'appareil est néanmoins lourd et nécessite l'installation d'un système de positionnement sous-marin de type USBL. Les opérations avec ce type de sonar ne sont donc valables que sur des superficies conséquentes.

\section{Référence}

IXSEA (site web). http://www.ixsea.com

IXSURVEY (site web). http://www.ixsurvey.com 